\title{
Análisis de la resolución de un problema de cinemática mediante el mapa conceptual híbrido
}

\section{Analysis of the resolution of a kinematics problem using the hybrid conceptual map}

\author{
Nehemías Moreno Martínez \\ Facultad de Ciencias, Universidad Autónoma de San Luis Potosí, San Luis Potosí, México \\ nehemias.moreno@uaslp.mx \\ Luis Enrique Hernández Zavala \\ Centro de Investigación y de Estudios Avanzados del Instituto Politécnico Nacional, Ciudad de México, México \\ luisenri.hernadez@cinvestav.mx \\ Eduardo Carlos Briceño Solís \\ Unidad de Matemáticas, Universidad Autónoma de Zacatecas, Zacatecas, México. \\ ecbs74@gmail.com
}

RESUMEN • El mapa conceptual híbrido se interpreta a partir de una adaptación a la física escolar de algunos elementos del enfoque ontosemiótico. La interpretación permite analizar la actividad fisicomatemática involucrada en la resolución de un problema físico. Mediante un estudio de caso descriptivo, la interpretación permitió estudiar las prácticas de resolución de un problema de movimiento parabólico realizadas por una profesora y dos de sus estudiantes de física universitarios. La interpretación permite analizar gráficamente la organización y conexión de los objetos fisicomatemáticos, notar algunos procesos involucrados en la resolución del problema y describir de manera más adecuada el operativismo ciego al interpretar la representación de los objetos fisicomatemáticos desde dos perspectivas, cognitiva/epistémica y ostensiva/no ostensiva, en lugar de interpretarla como interna/externa.

PALABRAS CLAVE: Herramienta gráfica; Mapa conceptual híbrido; Resolución de problemas; Movimiento parabólico; Física escolar.

ABSTRACT - The hybrid conceptual map is interpreted from an adaptation to school physics of some elements of the ontosemiotic approach. Interpretation allows to analyze the physical-mathematical activity involved in solving a physical problem. Through a descriptive case study, the interpretation made it possible to study the practices of solving a parabolic motion problem carried out by a teacher and two of her university physics students. The interpretation allows to graphically analyze the organization and connection of the physical-mathematical objects, to notice some processes involved in the resolution of the problem and to describe in a more adequate way the blind operativism when interpreting the representation of the physical-mathematical objects from two perspectives, cognitive/ epistemic and ostensive/non-ostensive, rather than interpreting it as internal/external.

KEYWORDS: Graphic tool; Hybrid conceptual map; Problem solving; Parabolic motion; School physics.

Recepción: octubre 2019• Aceptación: diciembre 2020

Moreno Martínez,N., Hernández Zavala, L. E. y Briceño Solís, E. C. (2021). Análisis de la resolución de un problema de cinemática mediante el mapa conceptual híbrido. Enseñanza de las Ciencias, 39(3), 157-176. 


\section{INTRODUCCIÓN}

La resolución de problemas en la clase de física es una actividad importante en el aprendizaje y evaluación de los conocimientos de los alumnos. Al resolver un problema, los docentes consideran su estructura, mientras que los estudiantes atienden aspectos superfluos (Buteler, Gangoso, Brincones y González, 2001) o construyen estructuras propias al resolverlo y, en el caso de ser congruentes con su conocimiento previo, evitan contrastar con la teoría física (Guisasola, Ceberio y Zubimendi, 2003).

Proporcionar pautas a los estudiantes para reflexionar sobre la resolución de problemas y la aplicación de sus conocimientos los ayuda a entender mejor los procesos de resolución y la manera en que aprenden (Hinojosa y Sanmartí, 2016). Concari y Giorgi (2000), al analizar los problemas resueltos en textos de física en cursos de universidad, advierten que estos muestran una visión alejada de lo que es el trabajo científico y sugieren al docente algunas estrategias de enseñanza y evaluación.

También se ha usado la V de Gowin (Escudero y Moreira, 1999) para promover el aprendizaje significativo mediante la interacción entre el conocimiento del alumno, los elementos conceptuales y lo que él podría realizar al resolver el problema (Gil, Solano, Tobaja y Monfort, 2013). La V se apoya en la noción de representación interna/externa, y la interna se refiere a los procesos de razonamiento y modelos mentales de las personas, las cuales, al explicitarse mediante distintos sistemas representacionales (escritura, representación matemática, entre otros) dan lugar a la representación externa (Aguilar, 2004). Mediante la $\mathrm{V}$ se ha modelado el operativismo ciego, el cual es descrito como una práctica procedimental donde se memorizan y archivan fórmulas prescindiendo de la interacción entre el dominio conceptual y procedimental (Escudero y Moreira, 1999).

Cabe señalar que, en el campo de la matemática educativa, se ha reportado que el tándem representación interna/externa es ambiguo para la descripción de la actividad matemática y en su lugar se ha propuesto usar las facetas duales o perspectivas ostensivo/no-ostensivo y cognitivo/epistémico (Font, Godino y D'Amore, 2007).

En el contexto de la física escolar, en el presente trabajo se plantea el problema de que la perspectiva interna/externa también podría desencadenar ciertas ambigüedades en el análisis de la actividad fisicomatemática. La representación interna/externa en la física escolar permite a los alumnos observar y distinguir diversos elementos epistemológicos que intervienen en la construcción de nuevos conocimientos (Escudero y Moreira, 1999); sin embargo, esto no dice nada acerca de cuál sería la representación interna correspondiente a dichos elementos epistemológicos representados externamente. También, en este sentido, la descripción del operativismo ciego mediante la V de Gowin queda en entredicho.

En esta investigación se planteó el objetivo de considerar las facetas duales ostensivo/no-ostensivo y cognitivo/epistémico, mediante la interpretación del mapa conceptual híbrido $(\mathrm{MCH})$ o mapa híbrido desde una adaptación del enfoque ontosemiótico (EOS), en el contexto de la física escolar, para analizar la resolución de un problema de movimiento parabólico por parte de una docente y sus alumnos de nivel universitario. El MCH, combinación del mapa conceptual con la técnica del diagrama de flujo, ha sido interpretado con el EOS para analizar un fragmento de una clase de matemáticas (Bencomo, Godino y Wilhelmi, 2004) y para investigar la enseñanza y el aprendizaje de las matemáticas mediante la resolución de problemas (Moreno, Torres y Zúńiga, 2019).

En otros trabajos, el $\mathrm{MCH}$ ha sido interpretado desde una adaptación incipiente del EOS a la física escolar (Moreno, Angulo, Reducindo y Aguilar, 2018; Moreno, Zúñiga y Tovar, 2018; Moreno, Aguilar, Angulo y Ramírez, 2019). En este trabajo se avanza con la adaptación del EOS para la física escolar y en su aplicación con la interpretación del $\mathrm{MCH}$. Tomando en cuenta que la construcción de una idea o noción física se realiza a partir de sucesivas búsquedas de semejanzas y diferencias con lo que el estudiante conoce, para luego hacer una generalización (Alomá y Malaver, 2007), el MCH se usa como 
una ayuda gráfica para estudiar la construcción del conocimiento físico a partir de las conexiones entre los objetos físicos y fisicomatemáticos que intervienen en la resolución de un problema.

\section{UNA APROXIMACIÓN TEÓRICA PARA LA FÍSICA ESCOLAR}

En esta sección se describen, en un primer momento, los elementos teóricos del EOS que fueron adaptados al contexto de la física escolar y, por último, se describe en qué consiste dicha adaptación.

\section{Algunos elementos teóricos del enfoque ontosemiótico}

Según el enfoque ontosemiótico (EOS), cuando un sujeto (novato o experto) resuelve una situaciónproblema, este lleva a cabo un sistema de prácticas donde participa un conjunto de objetos matemáticos primarios (lenguaje, conceptos, propiedades, procedimiento y argumentos). En una práctica, entendida como toda actuación realizada por el sujeto al resolver el problema (Godino, Batanero y Font, 2007), pueden emerger objetos matemáticos que son empleados por otras prácticas que conforman el sistema de prácticas.

El EOS señala que el sujeto realiza procesos cognitivos (entendidos en la forma entrada, proceso y producto) sobre los objetos matemáticos. La entrada es un objeto en una de sus facetas y, a través del proceso, se obtiene como producto el objeto en una faceta distinta. Algunos de los procesos son: $i$ ) idealización, que permite ir de un objeto observable como un signo o expresión algebraica (faceta ostensiva) a un objeto pensado (faceta no ostensiva); ii) materialización, que es inverso a la idealización y permite materializar o hacer público un objeto pensado (Font y Contreras, 2008); iii) particularización, donde un objeto general (faceta intensiva), por ejemplo, la familia de funciones, se usa de manera específica (faceta extensiva), por ejemplo, la función ; iv) representación, que permite atribuir un significante (faceta expresión) a un significado (faceta contenido); $v$ ) significación, donde a un significante se le atribuye un significado. La noción función semiótica relaciona los procesos, y iv) al considerar que los objetos matemáticos primarios pueden desempeñarse como contenido de dicha función.

Además de los procesos anteriores, el EOS considera otros procesos, como la argumentación, implicada en la generación de argumentos para sustentar procedimientos, y la algoritmización, relacionada con la realización de cierto procedimiento, entre otros.

\section{Una adaptación del enfoque ontosemiótico para la física escolar}

La aproximación teórica para la física escolar que se propone en este trabajo se apoya en la noción de objeto del interaccionismo simbólico (Blumer, 1969), entendido como todo aquello que puede ser indicado o señalado. En la física escolar se hace referencia a objetos físicos y a objetos fisicomatemáticos. Los objetos físicos son vistos como abstracciones o idealizaciones de los hechos, objetos concretos o entidades físicas, los cuales están vinculados a los resultados experimentales o la experiencia. No se trata de objetos ficticios como ocurre con los objetos matemáticos primarios del EOS. El objeto físico parte de la experiencia sensible, sin embargo, coincidimos con Herrero (2012) en que estas no son meras impresiones sensoriales, más bien son elaboraciones ordenadas objetivas susceptibles de formalización matemática.

En la actividad fisicomatemática, además de los objetos matemáticos primarios, también participan objetos físicos. Se propone agregar a los objetos matemáticos primarios una tipología de objetos físicos y, por otro, extender la noción de función semiótica, exclusiva de los objetos matemáticos en el EOS, para establecer relaciones de significación entre los objetos matemáticos primarios y los objetos físicos. Esto conduce a un nuevo conjunto de objetos, los objetos fisicomatemáticos. 
Debido a la noción de objeto empleado, se adopta una postura pragmatista de los objetos físicos y, por tanto, de los objetos fisicomatemáticos, pues se soslaya la naturaleza, la constitución material y el origen de los hechos físicos, de los objetos concretos o entidades físicas. Más bien, se da importancia a la practicidad o al uso de dichos objetos como herramientas para la resolución de problemas, por ejemplo, para realizar predicciones o descripciones de los fenómenos.

A continuación, se realiza una descripción de los objetos fisicomatemáticos y de los objetos físicos que intervienen en la actividad fisicomatemática:

i) Situación fisicomatemática problematizada: fenómenos físicos problematizados de manera cuantitativa, por ejemplo, el cálculo de la velocidad final de una partícula después de una colisión, que requiere de la interpretación física de los resultados. Situación física problematizada: fenómenos que se plantean de forma demostrativa y donde se cuestiona acerca de las creencias, por ejemplo, el prisma de Newton para mostrar la descomposición de la luz.

ii) Lenguaje fisicomatemático: se refiere al lenguaje matemático interpretado o significado en términos de los objetos físicos, por ejemplo, las «flechas» que aluden al vector fuerza. Lenguaje físico: representaciones pictóricas, esquemas o dibujos que aluden al mundo físico.

iii) Conceptos fisicomatemáticos: se enuncian mediante definiciones que son matematizadas y representadas por medio fórmulas, por ejemplo, "fuerza», entre otras. Conceptos físicos: enunciados que no se matematizan, tales como partícula, los dispositivos usados en el laboratorio, por ejemplo, la lente, el polarizador, la placa de pruebas (protoboard), entre otros, los cuales son definibles y ayudan a acceder a los conceptos físicos matematizables.

iv) Propiedades fisicomatemáticas: se refiere a enunciados acerca de los conceptos fisicomatemáticos que se pueden plantear matemáticamente, por ejemplo, el principio de Arquímedes para el concepto de fuerza de flotación. Propiedad física: enunciados que no se plantean matemáticamente, por ejemplo, la propiedad de que la luz se propaga en línea recta, si bien la representación pictórica de un rayo de luz es útil para pensar en la luz, pero dicha propiedad no tiene una formulación matemática.

v) Procedimiento fisicomatemático: se refiere al tratamiento o técnicas de cálculo interpretables desde el mundo físico, por ejemplo, la resolución de una ecuación de cinemática. En el laboratorio también se realiza un procedimiento físico que no se matematiza, por ejemplo, el armado de los dispositivos experimentales o la realización de mediciones experimentales.

vi) Argumentos fisicomatemáticos: se trata de enunciados que se emplean para validar o explicar el procedimiento o algoritmo de resolución de la situación física problematizada, por ejemplo, al resolver una ecuación cinemática que involucra la posición de una partícula en función del tiempo «t», algunas veces se obtiene una solución «t<0» y se argumenta descartarla al no tener sentido o significado físico en el contexto del problema que se está resolviendo. Argumentos físicos, empleados para justificar o explicar los procedimientos físicos y fisicomatemáticos, por ejemplo, cuando se experimenta con el péndulo, se argumenta que se debe medir la longitud del péndulo del punto de sujeción al centro de masa del cuerpo que pende, pues sobre este actúan las fuerzas.

La adaptación del EOS a la física escolar también considera otros elementos teóricos tales como prácticas, sistemas de prácticas, procesos y facetas. Cuando un sujeto resuelve una situación fisicomatemática problematizada este lleva a cabo un sistema de prácticas donde participan objetos físicos y objetos fisicomatemáticos. En la realización del sistema de prácticas el sujeto lleva a cabo ciertos procesos cognitivos (idealización, materialización, particularización, por mencionar algunos) que le permiten mirar a los objetos físicos y fisicomatemáticos desde distintas facetas (ostensivo/no-ostensivo, extensivo/intensivo, cognitivo/epistémico, entre otras). Además de los procesos anteriores, en la resolución 
de problemas planteados textualmente, la adaptación teórica también se apoya en otros procesos como el de comprensión lectora, el cual consiste en la realización de procesos como el de i) inferencia, que le permite relacionar las ideas del texto con sus propias ideas, plantear hipótesis, sacar conclusiones, entre otras, ii) memoria, para recuperar información u organizar su conocimiento, y iii) metacognición, que le permite organizar, cuestionar, planear, monitorear la comprensión, entre otros (Montealegre, 2004).

En el EOS, la actividad matemática induce la emergencia de objetos secundarios que son nuevamente ficticios, mientras que, con la adaptación del EOS a la física escolar, los objetos emergentes de la actividad fisicomatemática podrían tener un significado con algún elemento del mundo físico. La adaptación del EOS a la física escolar se apoya en la semiótica pragmática del EOS, es decir, el significado de los objetos físicos y fisicomatemáticos viene dado por el uso o función que dichos objetos tienen en el lenguaje, en el sentido de Wittgenstein (Godino, 2003). Es decir, los conceptos y las propiedades físicas y fisicomatemáticas son interpretados en el mismo sentido de los objetos matemáticos del EOS, esto es, como reglas gramaticales del uso de símbolos y expresiones, las cuales, en el caso de la física, no son reglas inventadas y tienen que ver con regularidades (patrones) en el mundo empírico y matemático.

\section{PROBLEMA Y METODOLOGÍA}

En este trabajo se aborda el problema de cómo una docente y sus estudiantes organizan y conectan los objetos físicos y fisicomatemáticos al enfrentarse a la tarea de resolver un problema de movimiento parabólico. Para abordarlo se interpretó y analizó el MCH desde la adaptación del EOS a la física escolar. Para el análisis se construye el $\mathrm{MCH}$ a partir de la producción escrita y la transcripción del discurso oral del sujeto que resuelve el problema de movimiento parabólico. El $\mathrm{MCH}$ es una representación ostensiva de los objetos físicos y fisicomatemáticos que fueron pensados (no ostensivos), organizados, conectados y luego materializados por el sujeto al resolver el problema. Desde la adaptación del EOS a la física escolar puede decirse que el $\mathrm{MCH}$ es de tipo epistémico si corresponde a la producción docente (experto) o bien cognitivo, si es construido de lo que produjo un estudiante (inexperto).

Se llevó a cabo una metodología cualitativa a través de un estudio de caso de tipo descriptivo ya que, por un lado, describe de manera secuencial la producción oral y escrita de los sujetos al resolver una situación problematizada de movimiento parabólico y, por otro lado, dicha producción fue representada de manera gráfica mediante el $\mathrm{MCH}$, lo que permitió observar, en el tiempo, los distintos objetos fisicomatemáticos, así como la organización y conexión entre ellos en un sistema de prácticas.

El estudio también fue interpretativo ya que, una vez elaborado el $\mathrm{MCH}$, fue analizado desde la adaptación del EOS. La comparación entre el MCH epistémico, correspondiente a la producción de una docente, y el MCH cognitivo a la producción de dos de sus estudiantes de física permitió conocer diferencias y semejanzas de los significados, los objetos empleados, las conexiones entre los objetos y los procesos cognitivos realizados.

Los participantes fueron una docente y dos de sus estudiantes denotados como Ana y Bruno. Los estudiantes de ingeniería fueron seleccionados aleatoriamente a partir de un grupo de 48 que cursaban la asignatura «Física A-Cinemática» de la Universidad Autónoma de San Luis Potosí, en México.

\section{Diseño de la actividad y herramienta de recolección de datos}

La tarea planteada a la docente y alumnos es una adaptación del problema de movimiento parabólico propuesto en el libro de Serway y Jewett (2008), el cual consiste en la descripción textual de la situación física del movimiento de un proyectil lanzado desde lo más alto de un edificio y el planteamiento de tres preguntas. La adaptación consistió en eliminar la última pregunta, de manera que solo solici- 
taba calcular el alcance desde la base del edificio y la altura donde fue lanzada la pelota. También se agregó una figura de la situación y un plano cartesiano "vs " para indicar el marco de referencia desde el cual se quiere resolver el problema (figura 1). El problema planteado fue:

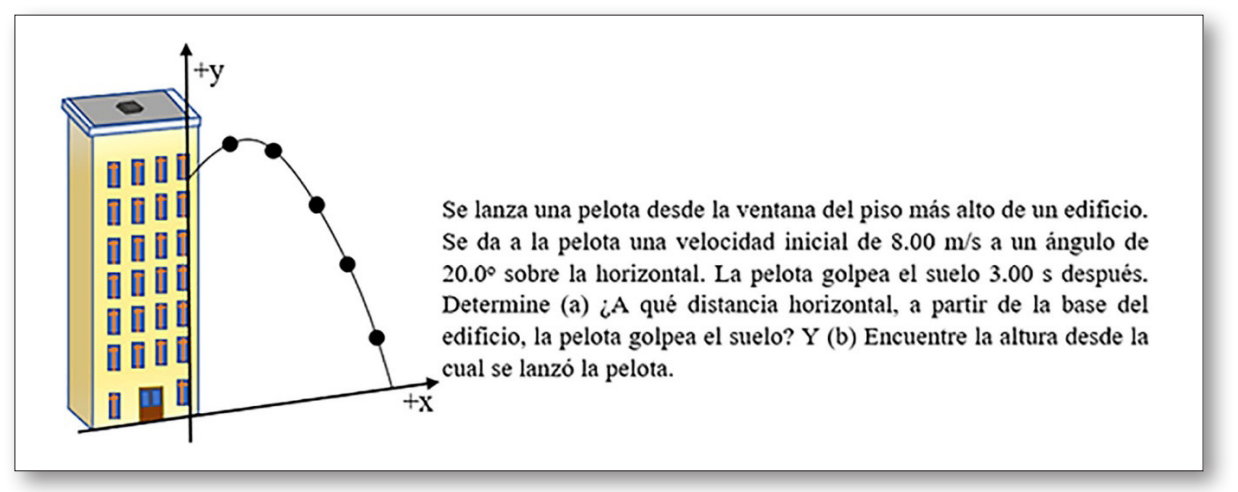

Fig. 1. Situación física problematizada de movimiento parabólico de un proyectil. Adaptado de Serway y Jewett (2008).

De manera individual se solicitó a la docente y estudiantes resolver en hojas blancas la situación física problematizada y, una vez hecho esto, se les pidió que resolvieran nuevamente el problema solo que esta vez explicando en voz alta el proceso de resolución y usando una pluma electrónica, Smart Pen Livescribe, que registra en tiempo real la producción oral y escrita de forma sincronizada al resolver el problema.

En la resolución del problema no se proporcionaron las fórmulas de cinemática, de manera que las empleadas en sus soluciones venían de su conocimiento previo. La docente resolvió el problema en dos minutos, Ana en siete minutos y Bruno en aproximadamente cuatro minutos.

Con el archivo electrónico generado por la pluma electrónica, se obtuvo la transcripción oral y la imagen de escritura, donde se identificaron las prácticas realizadas, los objetos fisicomatemáticos implicados y la organización de dichos objetos en las prácticas correspondientes. También fue posible advertir la realización de algunos procesos cognitivos comunes entre los sistemas de prácticas realizado por los sujetos investigados.

\section{Elaboración e interpretación del $\mathrm{MCH}$}

De acuerdo con la dualidad cognitiva/epistémica, el $\mathrm{MCH}$ es de tipo cognitivo cuando corresponde a la producción de un inexperto y de tipo epistémico cuando corresponde a la producción de un experto. La elaboración de ambos tipos de mapas se realiza mediante el proceso que se describe a continuación.

La figura 2 ilustra la producción de la docente al resolver el problema del movimiento parabólico. El texto en los recuadros muestra la transcripción del discurso oral y el texto en el centro presenta la producción escrita. Los recuadros se numeraron del 1 al 11 para describir la secuencia de resolución del problema y las flechas indican la parte del registro escrito que la docente explicó oralmente. Los recuadros contienen argumentos justificativos del procedimiento empleado y también propiedades fisicomatemáticas. Los enunciados pueden verse como la conexión, mediante palabras enlace, de los conceptos físicos y fisicomatemáticos que fueron marcados en negritas y subrayados en la figura 2 . Por ejemplo, en el recuadro (1), el argumento "Primero te dice la velocidad inicial que es $v_{0}=8 \mathrm{~m} / \mathrm{s}$ » está formado por dos representaciones del concepto velocidad inicial, las palabras «velocidad inicial» y los símbolos « $v_{0}=8 \mathrm{~m} / \mathrm{s}$, los cuales se conectan mediante las palabras enlace "primero te dice la» $\mathrm{y}$ «que es». 


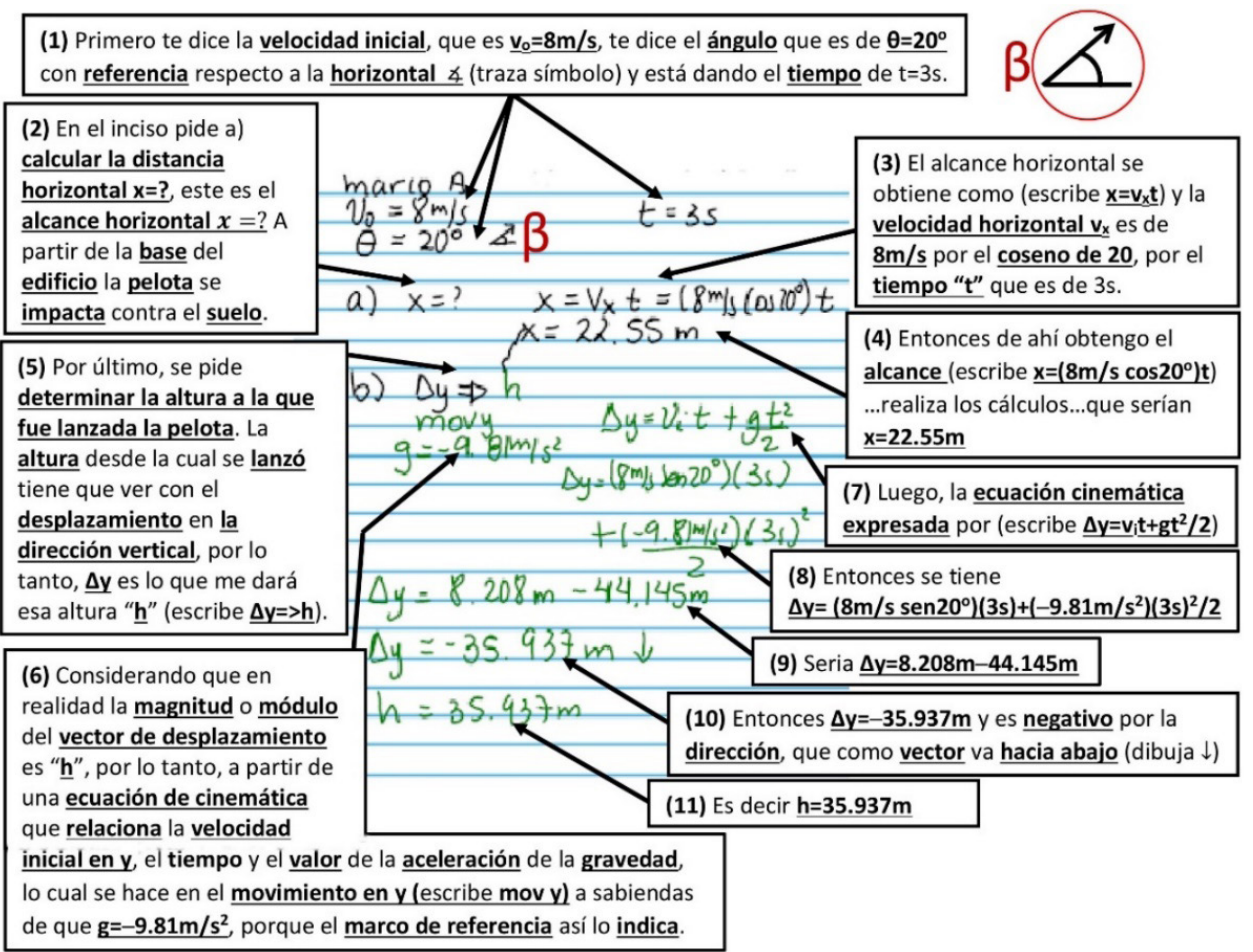

Fig. 2. Transcripción del discurso oral (recuadros) y registro escrito de una docente al resolver un problema de movimiento parabólico (elaboración propia).

En la figura 2 se observa que la docente resuelve el problema mediante tres prácticas, la primera de tipo interpretativa, ver recuadro (1); la segunda, recuadros (2), (3) y (4), determina la distancia horizontal recorrida por el proyectil; y la tercera, recuadros del (5) al (11), determina la altura desde la cual fue lanzada la pelota.

La construcción del MCH inicia con la descripción del sistema de prácticas, la cual consiste en «describir el movimiento de la pelota mediante el alcance y la altura desde la que fue lanzada del edificio», ver «Sp» en la figura 3. La Sp consta de tres prácticas, A, B y C, tituladas «Interpretar la situación-problema», "Calcular la distancia horizontal $\mathrm{x}=$ ?» $\mathrm{y}$ «Determinar la altura a la que fue lanzada la pelota», respectivamente. La primera práctica se coloca del lado izquierdo y las siguientes prácticas se colocan horizontalmente hacia la derecha.

Los recuadros de la figura 2 son representados en el $\mathrm{MCH}$, considerando los objetos (conceptos o propiedades expresados mediante palabras, símbolos o fórmulas) y las palabras enlace. Los objetos son puestos en recuadros que contienen otros parámetros para ayudar a la lectura e interpretación del $\mathrm{MCH}$, por ejemplo, un parámetro, representado mediante una secuencia de números enteros, se usa para referirse al orden en el que aparecen los objetos fisicomatemáticos en el discurso oral y escrito del sujeto.

De manera arbitraria, la numeración se repite en cada práctica, aunque podría no repetirse. Otro parámetro es el tiempo expresado en minutos y segundos, que puede ser obtenido cuando se reproduce el archivo digital (generado por la pluma electrónica) en la computadora. 
Un mismo objeto en el MCH puede presentar dos tiempos distintos. Esto se debe a que el sujeto se ha referido al mismo objeto en dos tiempos distintos en la resolución del problema. También puede suceder que dos objetos numerados consecutivamente posean el mismo tiempo, y esto es porque el registro de audio es de minutos y segundos, de manera que el sujeto podría decir dos palabras distintas en un mismo segundo.

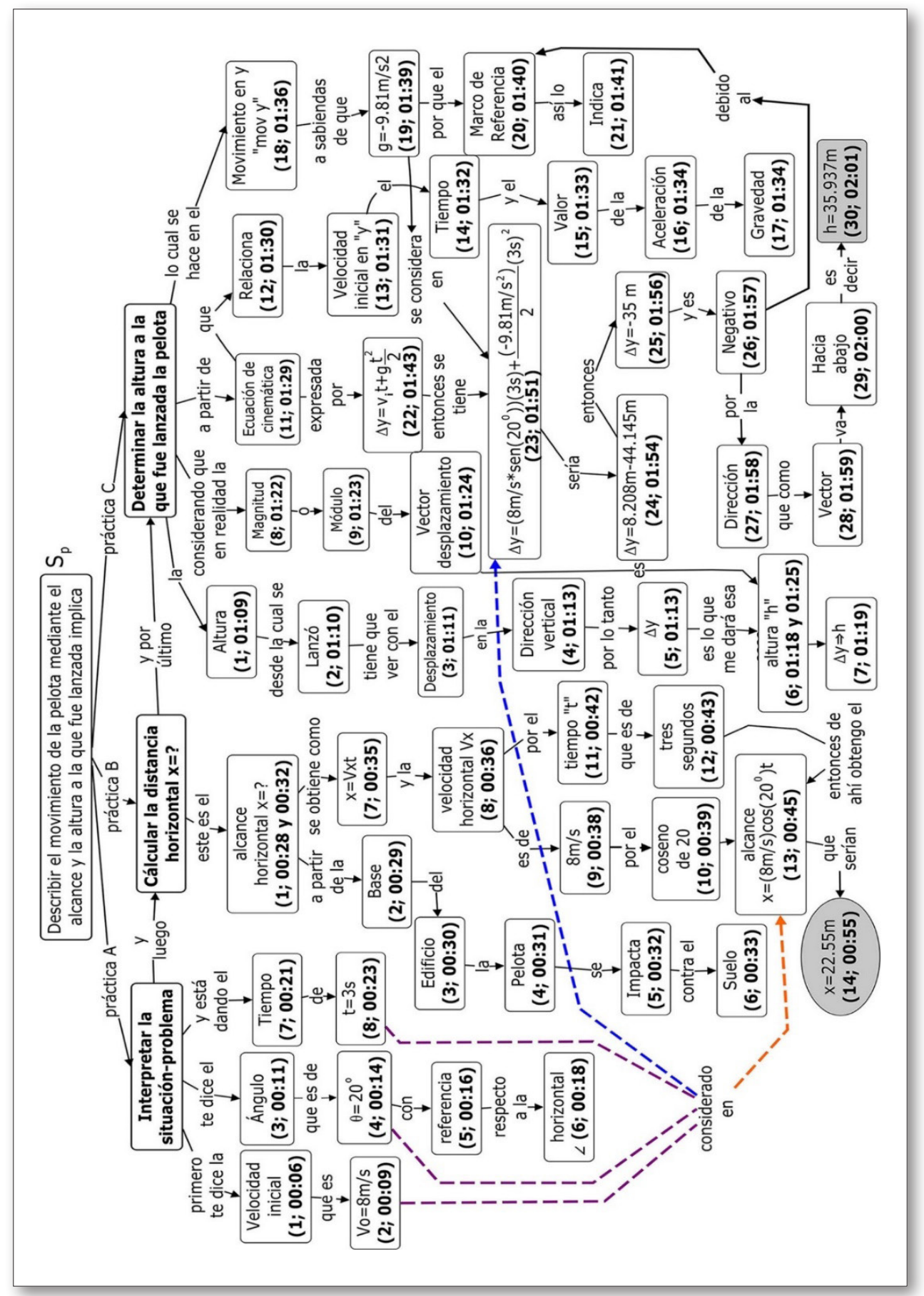

Fig. 3. MCH epistémico del sistema de prácticas realizado por una docente al resolver el problema de movimiento parabólico (elaboración propia). 
De acuerdo con el recuadro (1) de la transcripción, figura 2, la docente llevó a cabo una práctica A donde planteó tres argumentos: «Primero te dice la velocidad inicial, que es $v_{0}=8 \mathrm{~m} / \mathrm{s}$, «te dice el ángulo que es de $\theta \neq 20^{\circ}$ con referencia respecto a la horizontal Ł (traza símbolo de ángulo)»y «y está dando el tiempo de $\mathrm{t}=3 \mathrm{~s}$ ». El primer argumento se representa en el $\mathrm{MCH}$ mediante la ruta de lectura (1;00:06)-(2), el segundo como (3;00:11)-(4)-(5)-(6) y el tercero mediante (7;00:21)-(8). A partir de ahora se hará referencia a las cadenas de conceptos en el $\mathrm{MCH}$, en el que toda la producción de la figura 2 se encuentra representada en el $\mathrm{MCH}$ de la figura 3.

La materialización de (1;00:06)-(2) da cuenta del proceso de comprensión lectora del texto, ya que la docente infirió una relación entre el texto «se da a la pelota una velocidad inicial de $8.00 \mathrm{~m} / \mathrm{s}$ y su conocimiento previo velocidad inicial manifestado a través del empleo del símbolo « $v_{0}$ " en $(2 ; 00: 09)$ que no proviene del texto. También realizó un proceso de significación al atribuir al concepto (1;00:06) el significante (2;00:09).

En el caso del ángulo, la docente repite los procesos anteriores cuando materializa (3;00:11)(4;00:14). Por otro lado, mediante la idealización, la curva que representa la trayectoria de la pelota, la pelota, el edificio y el suelo fueron desmaterializados para ser analizados por la docente y, mediante la visualización, ella visualizó el ángulo formado entre la horizontal y la dirección de la velocidad inicial para luego materializarlo a través de (4;00:14)-(5)-(6; 00:18), figura 3, y « $\beta$ » en la figura 2 . El símbolo materializado en « $\beta$ » es un significante ostensivo del concepto no ostensivo ángulo. Para el caso del tiempo, la ruta (7;00:21)-(8) arroja evidencia de los procesos de comprensión lectora, particularización, significación y materialización.

En la práctica B, la ruta (1;00:28)-(2)-(3)-(4)-(5)-(6;00:32), del recuadro (2) de la figura 2, da evidencia del proceso de comprensión de la docente del inciso (a), por ejemplo, el problema menciona «distancia horizontal» y la docente comentó $(1 ; 00: 28)$ «alcance horizontal». También el problema presenta "golpea el suelo», mientras que la docente señala (5;00:32)-(6;00:33) «impacta contra el suelo», lo cual muestra la interacción entre el texto y el conocimiento previo de la docente. Así mismo, esta ruta da cuenta de un proceso de visualización del alcance de la pelota.

En la práctica B la docente realizó otros tres procesos identificables, uno de argumentación que le permitió considerar una propiedad física que la docente no materializó (la propiedad de que la aceleración horizontal en un movimiento parabólico es nula), pero que la llevó a la materialización de la fórmula general (7;00:35) y la significación de variables que componen la fórmula, por ejemplo, al significante "x» le atribuyó el significado de alcance horizontal, al significante " $v_{x}$ ", el significado de velocidad horizontal y al significante «t» como el tiempo. Después, apoyada en la propiedad de aceleración horizontal nula (no materializada), ella realizó un proceso de particularización de la variable " $v_{x}$ ", al considerar las condiciones específicas del problema abordado, es decir, se apoyó en (2;00:09) y (4;00:14) para luego integrarlos en (13;00:45). Finalmente, considerando el tiempo mediante (11;00:42)-(12;00:43), la docente particularizó la fórmula general en (13;00:45) para luego realizar un proceso de algoritmización que le condujo al resultado correcto (14;00:55).

En la práctica C, recuadros del (5) al (11) figura 2, la docente realizó un proceso de argumentación que mostró evidencia de la realización del proceso de visualización, al enfocarse en el movimiento vertical de un tiro parabólico, y de comprensión lectora al inferir una relación entre el inciso (b) del problema y el conocimiento previo del docente sobre vectores. Por ejemplo, infirió y visualizó que la altura solicitada -véase (1;01:09)-(2;01:10) - tiene que ver con el desplazamiento de la pelota en la dirección vertical (3;01:11)-(4)-(5;01:13) y, consecuentemente, que el módulo del vector desplazamiento es la altura (9;01:23)-(10;01:24)-(6;01:25). También realizó el proceso de significación al dar significado el símbolo « $\Delta y »$ en $(5 ; 01: 13)$ con el concepto de altura en $(7 ; 01: 19)$.

Los aspectos anteriores llevan a la docente a realizar un proceso de argumentación al enunciar el argumento general (11;01:29)-(12)-(13)-(14)-(15)-(16;01:34)-(17;01:34), luego, al enfocarse en 
(16;01:34)-(17;01:34), inicia la particularización de dicho argumento mediante la visualización del marco de referencia y el sentido de sus ejes la lleva a enunciar el argumento (18;01:36)-(19)-(20)-(21) acerca de la aceleración de la gravedad, lo que le permite recurrir a su memoria para asignarle un valor a dicha constante (19;01:39) y también atribuirle un signo negativo apoyándose en las convenciones que conoce.

El argumento general, con la particularización anterior de la aceleración de la gravedad, es materializado en (22;01:43). En este instante, la docente realizó un proceso de significación al atribuir un significado a cada símbolo que constituye la fórmula general, por ejemplo, el significante « $v_{i}$ " significa la componente «y» de la velocidad inicial y el significante «» es interpretado como el tiempo. La particularización culmina en (23;01:51), donde también ha incorporado los objetos (2;00:09), (4;00:14) y (8;00:23) de la práctica A.

La docente llevó a cabo un proceso de algoritmización mediante el procedimiento (23;01:51)(24;01:54) para obtener el resultado (25;01:56); sin embargo, mediante el argumento (26;01:57)(27)-(28)-(29;02:00) logró significar el signo negativo obtenido en dicho resultado, lo que le permitió reescribir la solución correcta $(30 ; 02: 01)$.

Con el análisis anterior se observa que en el sistema de prácticas realizado por la docente aparece de manera recurrente, en cada práctica, un conjunto de procesos cognitivos (figura 4) responsable de la producción de conocimiento.

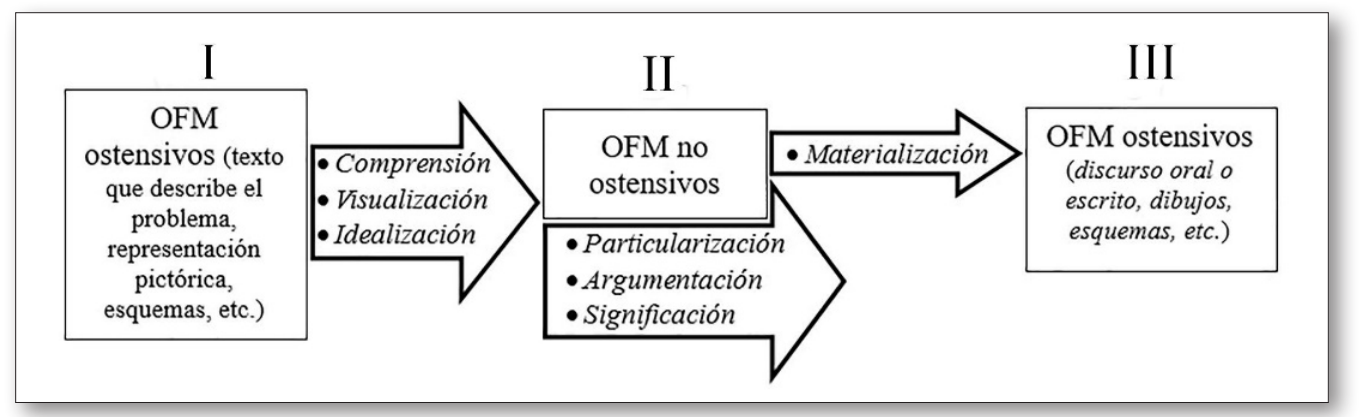

Fig. 4. Conjunto de procesos implicados en la producción del objeto físico matemático, OFM (elaboración propia).

El esquema de la figura 4 muestra la secuencia en la realización de los procesos antes mencionados. El texto y la figura que describen la situación problematizada proveen de un conjunto de objetos fisicomatemáticos (OFM) ostensivos I, los cuales son comprendidos (mediante la inferencia, la metacognición y la memoria), visualizados e idealizados para conformar un conjunto de OFM no ostensivos II. La comprensión lectora permite al sujeto establecer relaciones de inferencia entre los OFM del texto y los OFM provenientes del conocimiento previo del sujeto, también permite acceder a las fórmulas, datos, procedimientos, reglas o convenciones que se encuentran en la memoria del sujeto.

Algunos OFM no ostensivos en II son particularizados, otros son relacionados entre sí mediante funciones semióticas para establecer relaciones de significación y, mediante la argumentación, se establecen argumentos que sustentan o validan los procedimientos. Finalmente, algunos OFM en II son materializados o comunicados en III. 


\section{ANÁLISIS DE LOS MAPAS CONCEPTUALES HÍBRIDOS COGNITIVOS}

En esta sección se analiza la resolución del problema realizada por los alumnos; primero se analiza la resolución de Ana y posteriormente la de Bruno. Por cuestiones de espacio, únicamente se presentará el $\mathrm{MCH}$ de los alumnos y se realizará el análisis mediante la aproximación teórica desarrollada.

\section{El MCH de la alumna Ana}

El MCH de Ana (figura 5) muestra el uso de fórmulas de cinemática y la evaluación de la pertinencia de estas a partir de criterios operativos alejados de interpretaciones físicas. El argumento (1;00:04)-(2)(3)-(4)-(5)-(6) muestra que Ana realiza un proceso de comprensión lectora del problema; sin embargo, a diferencia del $\mathrm{MCH}$ epistémico, ella infiere una relación entre su conocimiento previo y aspectos superficiales del problema, como el número de incisos (3;00:11), y lo que se solicita calcular, la distancia horizontal (4;00:13) y la altura de lanzamiento (5;00:14)-(6).

Ana realizó el proceso de metacognición y memoria, ya que planeó resolver el problema al tener en cuenta fórmulas de tiro parabólico (7;00:23)-(8;00:29) que recuperó de su conocimiento previo; por ejemplo, materializó la fórmula para calcular la velocidad final en "y» mediante (9;00:34)-(10;00:38), la cual descartó al apoyarse en el argumento superficial (10;00:38)-(11)-(12). También materializó la fórmula para calcular la distancia en "y» a través de (13;00:47)-(14), pero no señaló ningún argumento de empleo. Ana, continuando con el plan del uso de fórmulas, materializó la fórmula para calcular la distancia máxima en «x» mediante (15;01:01)-(16)-(17;01:05), la cual descartó segundos después al argumentar, por un lado, que no era útil y, por otro al señalar la existencia de un despeje fácil, a través de (17;01:05)-(18)-(19)-(20;01:18).

Apoyado en argumentos anteriores y en el proceso de memoria, Ana recuperó información sobre el concepto de tipo parabólico $(22 ; 01: 25)$ que materializó al enunciar la propiedad de dos movimientos unidimensionales "x» e «y» a través de (21;01:23)-(22;01:25)-(23)-(24)-(25)-(26;01:33) y, también, al considerar la fórmula (28;01:36), que justificó al argumentar (27;01:35)-(28;01:36)-(29)-(30)(31;01:42). Es importante señalar que $(28 ; 01: 36)$ es un objeto emergente de la práctica que forma parte del plan elaborado para resolver el problema. También materializó la fórmula $(32 ; 01: 46)$ sin enunciar argumentos.

Posteriormente, Ana materializó el argumento (33;02:00)-(34:02:02) para señalar el «marco» donde se deben «extraer» los datos. Lo anterior da cuenta de la observación del marco de referencia dibujado en la representación pictórica que acompaña el problema y de la realización del proceso de comprensión lectora al relacionar las ideas del texto del problema con las ideas de Ana. Esto último llevó a Ana a significar y a materializar los datos numéricos que presenta el texto con los símbolos $\left(v_{0}, \angle \mathrm{y} t\right)$ presentes en el conocimiento previo de ella en (35;02:05)-(36), (37;02:11)-(38) y (39;02:15)-(40). Cabe señalar que (36;02:09), (38;02:13) y (40;03:17) también son objetos emergentes de esta primera práctica.

Asimismo, mediante el proceso de comprensión lectora, Ana materializó lo que el problema solicita calcular, es decir, la distancia horizontal $(41 ; 02: 18)-(42 ; 02: 19)$ y la altura desde la cual se lanzó la pelota $(41 ; 02: 18)-(43 ; 02: 21)$.

En la práctica B, en (1;02:41)-(2)-(3)-(4)-(5;03:04), Ana determinó la magnitud de las componentes de la velocidad inicial $v_{i x}$ y $v_{i y}$ al usar objetos emergentes $(36 ; 02: 09)$ y $(38 ; 02: 13)$, de la práctica A, en la particularización de las expresiones generales, no materializadas, de las componentes de un vector (vector $\vec{A}$ con componentes $A_{x}=A \cos \theta$ y $A_{y}=A \operatorname{sen} \theta$ ). También realizó el proceso de algoritmización, al realizar los cálculos, y el proceso de significación, al asociar los símbolos « $v_{i x}$ " $\mathrm{y}$ " $v_{i y}$ " de su conocimiento previo con los valores numéricos emergentes. Se descarta la realización del proceso de visualización por parte de Ana, pues no materializa ningún argumento relacionado con el ángulo de disparo del proyectil o el marco de referencia, más bien solo fue el uso de fórmulas. 


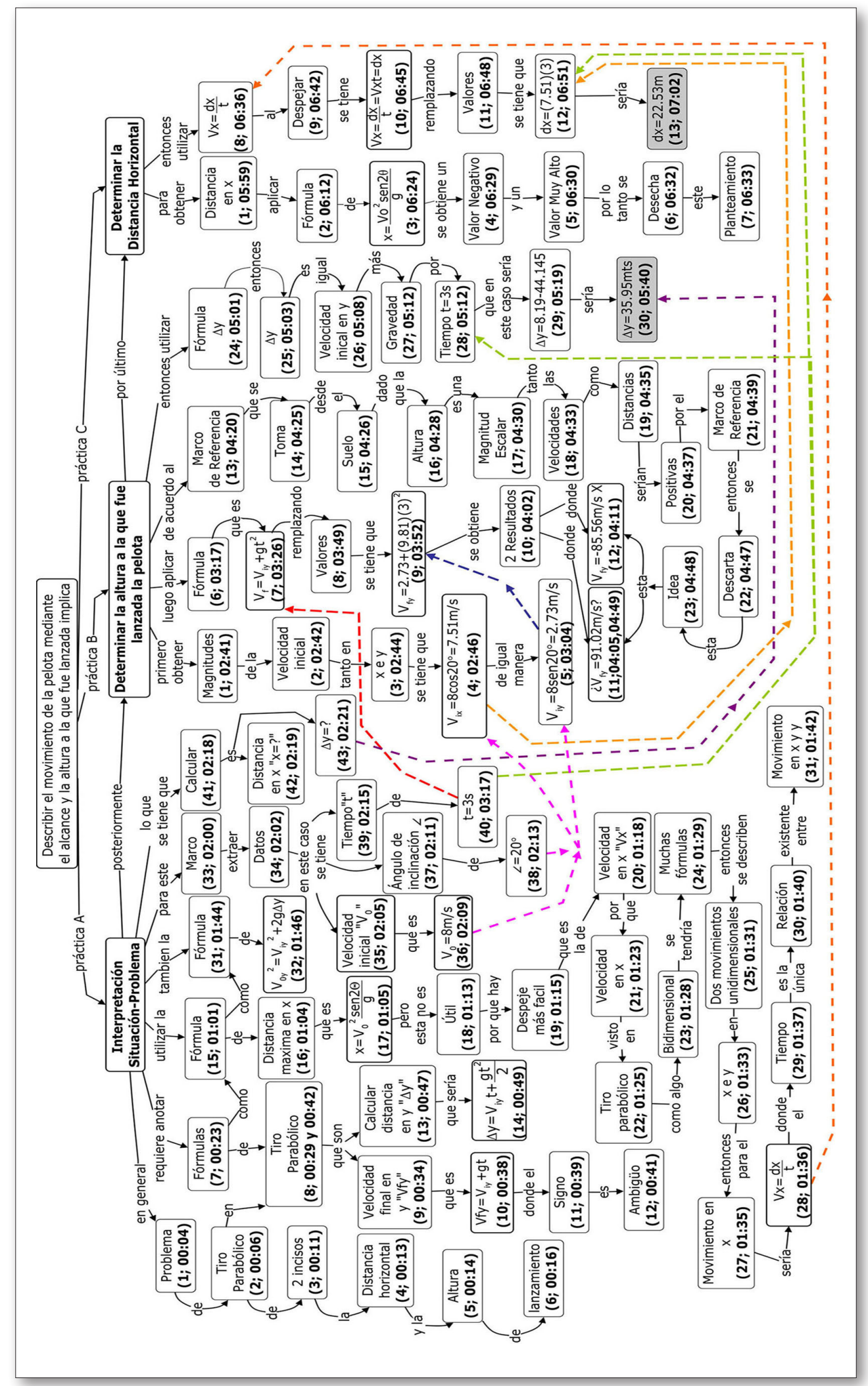

Fig. 5. MCH cognitivo correspondiente a la producción de Ana (elaboración propia). 
Posteriormente, Ana materializó una fórmula de cinemática (incorrecta) mediante (6;03:17)(7;03:26), que no había señalado en la práctica A. Luego particularizó dicha fórmula mediante (7;03:26)-(8)-(9), al sustituir la magnitud de la componente vertical de la velocidad inicial, (5;03:04)(9;03:52), el objeto emergente tiempo $(40 ; 03: 17)$ de la práctica A y el valor de la aceleración de la gravedad que recuperó de su conocimiento previo mediante el proceso de memoria. Ana realizó un proceso de algoritmización incorrecto, pues obtuvo dos soluciones a partir de (9;03:52), lo que refleja una nula metacognición del proceso realizado, en el sentido de que no cuestionó si lo que estaba realizando era correcto.

Cabe señalar en este punto que Ana llevó a cabo un operativismo ciego, pues si bien realizó los procesos de particularización y materialización en (9;03:52)-(10)-(11;04:05) y (12;04:11), no realizó adecuadamente otros procesos, por ejemplo, mediante el proceso de argumentación argumentó incorrectamente que «la altura es una magnitud escalar, tanto las velocidades y las distancias deben ser positivas» en (16;04:28)-(17)-(18)-(19)-(20;04:37), ya que "por el marco de referencia entonces se descarta esta idea» en (21;04:39)-(22)-(23), con lo cual rechazó las soluciones encontradas (12;04:11) y (11;04:49). Tampoco realizó el proceso de comprensión lectora correctamente, pues realizó incorrectamente el proceso de memoria al escribir de manera inapropiada la fórmula $(7 ; 03: 26)$ con la variable temporal al cuadrado.

Habiendo descartado los resultados anteriores, Ana buscó otra alternativa para determinar la altura desde la cual fue lanzada la pelota y materializó verbalmente una fórmula -véase (24;05:01)-(25)-(26)(27)-(28;05:12) - que había señalado pero no analizado en la práctica A mediante (14;00:49). En esta ocasión, Ana realizó los procesos de comprensión lectora (memoria), particularización (al considerar las condiciones específicas del problema en la fórmula general) y algoritmización de manera adecuada mediante (29;05:19)-(30;05:40), que llevaron a Ana al resultado correcto.

Por último, en la práctica $\mathrm{C}$, Ana materializó otra fórmula general para determinar la distancia horizontal de la base del edificio, hasta donde la pelota toca con el suelo, esto mediante (1;05:59)-(2)(3;06:24), sobre la cual realizó posteriormente los procesos de particularización, sustituyendo los objetos emergentes (36;02:09), (38;02:13) y (40;03:17) de la práctica A, y de algoritmización, con apoyo de la calculadora, para obtener un valor "negativo" $\mathrm{y}$ "muy alto" que enunció mediante el argumento (3;06:24)-(4)-(5;06:30), lo cual consideró como criterio suficiente para descartar dicho resultado mediante $(5 ; 06: 30)-(6)-(7 ; 06: 33)$.

Se trata de un operativismo ciego en el que, si bien es posible observar indicios de la realización de los procesos de particularización y materialización (3;06:24)-(4)-(5), no llevó a cabo de manera adecuada el proceso de memoria, al no considerar las condiciones que se requieren para poder usar la fórmula (3;06:24). Tampoco llevó a cabo el proceso de argumentación correctamente, al apoyarse en un argumento alejado de cualquier interpretación física. Cabe señalar que Ana también escribió incorrectamente la fórmula del alcance del proyectil (3;06:24).

Dado el resultado anterior, como alternativa, Ana materializó la fórmula (8;06:36), que había señalado en la práctica A como adecuada mediante la ruta (22;01:25)-(23)-(24)-(25)-...-(31;01:42). Ana llevó a cabo un proceso de significación adecuado al dotar de significado cada uno de los parámetros de la fórmula (8;06:36), luego realizó los procesos de algoritmización y materialización de manera adecuada a través de (8;06:36)-(9)-(10)-(11)-(12;06:51), que inició con el despeje de la distancia horizontal, posteriormente con la sustitución del valor de la componente «x» de la velocidad inicial (4;02:46), emergente de la práctica $\mathrm{B}$, y la sustitución del tiempo (39;02:15), emergente de la práctica A, para obtener el resultado correcto (13;07:02). Cabe señalar que en esta práctica Ana no se apoyó en ningún argumento para sustentar o validar el procedimiento realizado.

Ana obtuvo soluciones correctas al problema, sin embargo, atendió aspectos superfluos del problema (Buteler, Gangoso, Brincones y González, 2001) y construyó un mecanismo propio de resolu- 
ción sin interpretación física (Guisasola, Ceberio y Zubimendi, 2003). En cada práctica materializó fórmulas de manera consecutiva, las cuales fue descartando mediante criterios inadecuados (el signo es ambiguo, despeje más fácil o que las magnitudes físicas como la velocidad y las distancias deben ser positivas) hasta encontrar aquella fórmula, acorde con sus criterios, que le condujo a obtener los resultados correctos. Por otro lado, no se encontraron indicios de la realización de un proceso de visualización útil para resolver el problema, por ejemplo, respecto al marco de referencia, en la práctica A la alumna señaló el marco de referencia para indicar la extracción de datos del problema y, en la práctica B, empleó el marco de referencia como parte del criterio de velocidades y distancias positivas -véase (13;04:20)-(14)-...-(22)-(23;04:48) - para interpretar los resultados $(11 ; 04: 49)$ y $(12 ; 04: 11)$ de una fórmula incorrecta $(7 ; 03: 26)$.

\section{El MCH del alumno Bruno}

El sistema de prácticas realizado por Bruno se ilustra en el $\mathrm{MCH}$ de la figura 6. Con la primera práctica Bruno realizó un proceso de comprensión lectora, que le permitió inferir una relación entre el texto y su conocimiento previo acerca del lanzamiento de la pelota desde el edificio. Bruno visualizó los elementos que presenta la figura que acompaña el texto y materializó un nuevo marco de referencia en cuyo origen la pelota fue lanzada -véase (1;00:03)-(2)-(3)-(4)-(5;00:12)-. Posteriormente, él materializó una línea horizontal desde el origen del nuevo marco y visualizó el ángulo de disparo de la pelota, (5;00:12)-(6;00:18)-(7)-(8)-(9;00:28).

A continuación, Bruno realizó el proceso de memoria y recordó que ante el tipo de problema planteado se debe calcular la velocidad final en la dirección «», considerando la componente horizontal de la velocidad inicial y una aceleración; sin embargo, con esta idea Bruno mostró un desconocimiento de la propiedad física de que la aceleración horizontal es nula en el tiro parabólico, lo que le llevó a emplear la fórmula general ( $v_{f x}=v_{i x}+a_{x} t$, no materializada), que particularizó y materializó mediante (10;00:37)-(11)-(12)-(13)-(14)-(15;00:57) y a realizar el proceso de algoritmización (16;01:06) para obtener el resultado incorrecto $(17 ; 01: 09)$. Bruno mostró desconocimiento de la propiedad de aceleración horizontal nula y una significación inadecuada de los parámetros de la fórmula cinemática general, que le llevó a emplear la aceleración de la gravedad en los cálculos del movimiento en la dirección horizontal.

En la práctica B, Bruno realizó un proceso de comprensión lectora del primer inciso del problema y también visualizó el desplazamiento horizontal de la pelota, lo cual argumentó mediante (1;01:14)(2)-(3)-(4;01:19). Luego realizó el proceso de memoria y recordó que en un movimiento parabólico $(5 ; 01: 27)-(6 ; 01: 30)$ se requiere usar la fórmula que materializó mediante (7;01:33)-(8)-(9)-(10)$(11 ; 01: 46)$. 


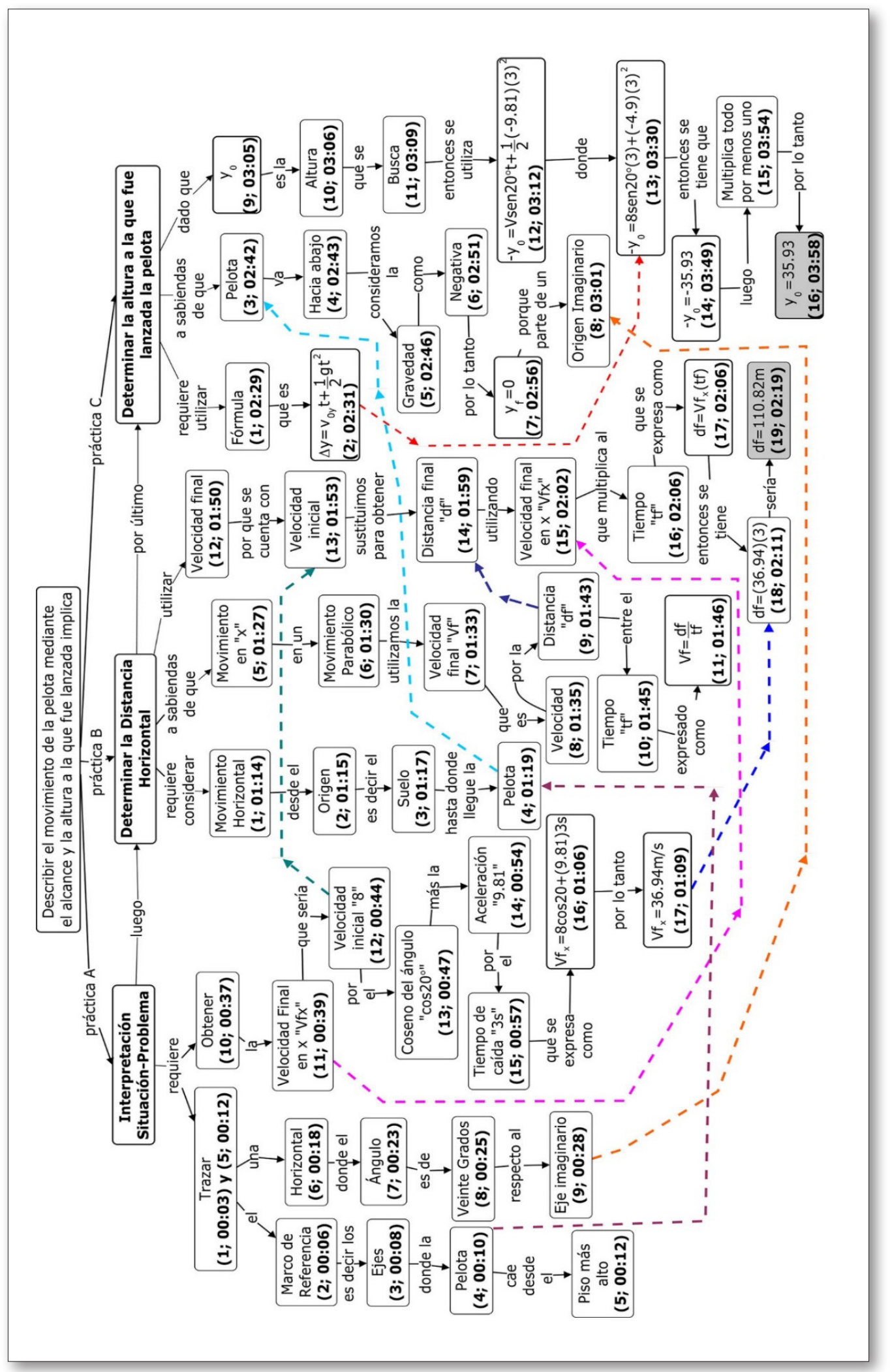

Fig. 6. MCH cognitivo correspondiente a la producción de Bruno (elaboración propia). 
Posteriormente realizó un procedimiento de despeje de la fórmula en (12;01:50)-(13)-(14)-(15)(16)-(17;02:06); luego realizó los procesos de particularización y significación que le permitieron sustituir los objetos emergentes (15;00:57) y (17;01:09) de la práctica A en la fórmula (17;02:06) de la práctica B; y a continuación, después de un proceso de algoritmización logró materializar (18;02:11) y el resultado incorrecto de la distancia final (19;02:19). Este resultado incorrecto muestra que el alumno atribuyó un significado inadecuado a los parámetros de la fórmula cinemática, en este caso, de $(17 ; 02: 06)$.

Finalmente, Bruno llevó adecuadamente la última práctica C, pues le permitió obtener un resultado correcto para el inciso (b) del problema. Realizó el proceso de memoria y recordó la fórmula general (1;02:29)-(2;02:31) y después visualizó la trayectoria de la pelota (3;02:42)-(4;02:43), así como el marco de referencia y el sentido de sus ejes, que lo llevó a realizar un proceso de argumentación para justificar el signo negativo en la aceleración de la gravedad (5;02:46)-(6), la condición final (7;02:56)(8) e inicial (9;03:05)-(10)-(11;03:09) de la trayectoria de la pelota.

En (12;03:12)-(13;03:30) Bruno llevó a cabo un proceso de particularización de la fórmula general (2;02:31), pues consideró las condiciones específicas de la situación abordada. También realizó un proceso de algoritmización al materializar el procedimiento que aparece en (12;03:12)-(13)-(14)(15;03:54) y que le llevó a obtener el resultado correcto (16;03:58).

En general, Bruno llevó a cabo el conjunto de procesos esquematizados en la figura 4 en las tres prácticas; sin embargo, en el caso de las prácticas A y B, el desconocimiento de la propiedad de aceleración horizontal nula y de la significación inadecuada de los parámetros de las fórmulas (16;01:06) y (17;02:06) le condujeron a resultados incorrectos.

Bruno no llevó a cabo un operativismo ciego, de hecho, el sistema de prácticas de él con 52 objetos o recuadros (igual que el número de objetos presentes en el mapa de la docente, figura 6) luce un poco más compacto que el de Ana con 74 objetos (figura 5). También cabe señalar que Bruno no materializó fórmulas de cinemática innecesarias, ni las descartó a partir de criterios inadecuados.

\section{COMPARACIÓN ENTRE EL MCH EPISTÉMICO Y EL MCH DE LOS ESTUDIANTES}

En la resolución del problema, la docente llevó a cabo un conjunto de procesos cognitivos (figura 4) que permitió la emergencia de nuevos objetos fisicomatemáticos en las prácticas. Los objetos emergentes en una práctica son empleados en otras permitiendo la conexión entre ellas con la emergencia de otros objetos. Cabe señalar que es necesaria la realización de dicho conjunto de procesos; por ejemplo, Ana no realizó el proceso de visualización y llevó a cabo un proceso de argumentación a través de criterios inadecuados, lo que la condujo a la realización de un operativismo ciego de resolución (Escudero y Moreira, 1999). Sin embargo, dicho conjunto de procesos podría no ser suficiente, por ejemplo, Bruno realizó dichos procesos en las prácticas $\mathrm{A}$ y $\mathrm{B}$, pero el desconocimiento de la propiedad de aceleración horizontal nula le llevó a obtener un resultado incorrecto.

La producción de la docente en la figura 2 y el MCH de la figura 3 pueden ser considerados como representaciones externas de las representaciones mentales de la docente, de hecho, si planteamos el mismo problema a otro profesor, se obtendría otra forma de resolverlo, pues los conocimientos fisicomatemáticos en distintos marcos institucionales adoptan formas particulares. La diferencia en las respuestas advierte distintas representaciones internas. De este modo, la representación externa es una manera de presentar diversos elementos epistemológicos en la construcción del conocimiento físico que supone un razonamiento único correcto en sentido absoluto, pues no señala cuál sería la representación institucional interna correspondiente. 
La ambigüedad anterior queda superada mediante el empleo de las facetas ostensivo/no-ostensivo y cognitivo/epistémico. La producción y el MCH obtenido de la resolución de un problema, pueden ser considerados como representaciones ostensivas del sistema de prácticas donde participan objetos físicos y fisicomatemáticos no ostensivos. Si el sujeto es la docente experta, se dice que la producción y el mapa son epistémicos, mientras que si se trata de alguno de los estudiantes son cognitivos.

Por otro lado, el MCH de Ana deja en entredicho el seńalamiento de Escudero y Moreira (1999) acerca del operativismo ciego, modelado mediante un sector de la V de Gowin que muestra la ausencia de relación entre los aspectos conceptuales y procedimentales. El $\mathrm{MCH}$, al mostrar con detalle las conexiones entre los objetos fisicomatemáticos que intervienen en la resolución del problema, permite observar que sí hay una interacción entre lo conceptual y procedimental, por ejemplo, la conexión entre (21;01:23)-(22)-...-(28;01:36) y (1;02:41)-(2)-(3)-(4;02:46) con el procedimiento (8;06:36)(9)-...-(13;07:02).

\section{CONCLUSIONES}

Las facetas ostensiva/no-ostensiva y cognitiva/epistémica, a través de la interpretación del MCH desde la adaptación del EOS a la física escolar, permitió analizar la actividad fisicomatemática implicada en la resolución del problema de movimiento parabólico por una docente y sus estudiantes.

La interpretación del $\mathrm{MCH}$ permite superar la ambigüedad que surge del manejo de la representación interna/externa en el análisis de la resolución de un problema físico, así se tiene que la producción, y por tanto el $\mathrm{MCH}$ correspondiente a ella, implicada en la resolución del problema, es de tipo epistémica o institucional, si esta corresponde a un docente experto, o bien es cognitiva, si corresponde a un estudiante. Al presentar la producción escrita y la transcripción del discurso oral, este es interpretado como una representación ostensiva del sistema de prácticas, es decir, se trata de una materialización de un conjunto de objetos físicos y fisicomatemáticos no ostensivos que fueron organizados y relacionados por el sujeto al resolver el problema. En contraste, la interpretación del MCH como una representación externa de diversos elementos epistemológicos del conocimiento físico soslaya la representación interna de la docente y obliga a pensar en un razonamiento absolutista, prescindiendo de las formas particulares que adopta el conocimiento fisicomatemático en los distintos marcos institucionales.

La realización de los procesos ilustrados en la figura 4 es una condición necesaria para la resolución del problema físico; sin embargo, no es suficiente, pues el desconocimiento de alguna propiedad física podría conducir a resultados incorrectos. La interacción entre el conocimiento nuevo que presenta el texto que describe el problema y el conocimiento previo del sujeto, puede ser modelado también mediante el esquema de la figura 4. En este sentido, prescindir del proceso de visualización y realizar de manera inadecuada el proceso de argumentación podría conducir al sujeto a la realización de un sistema de prácticas carente de elementos discursivos adecuados. La representación de los objetos físicos y fisicomatemáticos, y de sus conexiones mediante el $\mathrm{MCH}$, permiten mirar el operativismo ciego como una realización inadecuada o la ausencia de algunos de los procesos, en lugar de considerarla como una actividad netamente procedimental.

\section{REFERENCIAS BIBLIOGRÁFICAS}

Aguilar, T. M. F. (2004). El Mapa Conceptual: Un texto a interpretar. En A. J. Cañas, J. D. Novak y F. M. Gonzáles (Eds.), Concept Maps: Theory, Methodology, Technology (pp. 31-38). Pamplona, España: Dirección de Publicaciones de la Universidad Pública de Navarra. 
Alomá, C. E. y Malaver, M. (2007). Los conceptos de calor, trabajo, energía y teorema de Carnot en textos universitarios de termodinámica. Educere, 11(38), 477-488.

Bencomo, D., Godino, J. D. y Wilhelmi, M. R. (2004). Elaboración de redes ontosemióticas de configuraciones didácticas con Atlas/TI. En A. J. Cañas, J. D. Novak y F. M. González (Eds.), Proceedings of the First International Conference on Concept Mapping (pp. 71-74). Pamplona, España: Dirección de Publicaciones de la Universidad Pública de Navarra.

Blumer, H. (1969). Symbolic Interactionism, perspective and method. Upper Saddle River, NJ: Prentice Hall, Inc.

Buteler, L., Gangoso, Z., Brincones, C. I. y González, M. M. (2001). La resolución de problemas en física y su representación: Un estudio en la escuela media. Enseñanza de las Ciencias, 19(2), 285-295.

Concari, S. B. y Giorgi, S. M. (2000). Los problemas resueltos en textos universitarios de física. Enseñanza de las Ciencias, 18(3), 381-390.

D’Amore, B. y Godino, D. J. (2007). El enfoque ontosemiótico como un desarrollo de la teoría antropológica en didáctica de la matemática. Revista Latinoamericana de Investigación en Matemática Educativa, 10(2), 191-218.

Escudero, C. y Moreira, M. A. (1999). La V epistemológica aplicada a algunos enfoques en resolución de problemas. Enseñanza de las Ciencias, 17(1), 61-68.

Font, M. V. y Contreras, Á. (2008). The problem of the particular and its relation to the general in mathematics education. Educational Studies in Mathematics, 69(1), 33-52. https://doi.org/10.1007/s10649-008-9123-7

Font, M. V., Godino, D. J. y D'Amore, B. (2007). An onto-semiotic approach to representations in mathematics education. For the Learning of Mathematics, 27(2), 2-14.

Gil, J., Solano, F., Tobaja, L. M. y Monfort, P. (2013). Propuesta de una herramienta didáctica basada en la V de Gowin para la resolución de problemas de física. Revista Brasileira de Ensino de Física, 35(2), 2402-2414. http://dx.doi.org/10.1590/S1806-11172013000200017

Godino, D. J. (2003). Teoría de las funciones semióticas, Un enfoque ontológico-semiótico de la cognición e instrucción matemática (documento no publicado). Obtenido de https://www.ugr.es/-jgodino/ funciones-semioticas/monografiatfs.pdf

Godino, D. J., Batanero, C. y Font, M. V. (2007). The ontosemiotic approach to research in mathematics education. ZDM The International Journal on Mathematics Education, 39(1-2), 127-135. https://doi.org/10.1007/s11858-006-0004-1

Guisasola, J., Ceberio, M. y Zubimendi, J. L. (2003). El papel científico de las hipótesis y los razonamientos de los estudiantes universitarios en resolución de problemas de física. Investigaçóes em Ensino de Ciências, 8(3), 211-229.

Herrero, M. A. (2012). Instrumentalismo y realismo en la física de James C. Maxwell. Naturaleza y Libertad. Revista de Estudios Interdisciplinarios, 1(1), 77-138.

Hinojosa, J. y Sanmartí, N. (2016). Promoviendo la autorregulación en la resolución de problemas de física. Ciência \& Educação, 22(1), 7-22. https://doi.org/10.1590/1516-731320160010002

Montealegre, R. (2004). La comprensión del texto: sentido y significado. Revista Latinoamericana de Psicología, 36(2), 243-255.

Moreno, M. N., Aguilar, T. M. F., Angulo, V. R. G. y Ramírez, M. J. C. (2019). Análisis de la resolución de problemas de hidrostática en el bachillerato. Revista Electrónica de Enseñanza de las Ciencias, 18(1), 274-296. 
Moreno, M. N., Angulo, V. R. G., Reducindo, R. I. y Aguilar, P. R. M. (2018). Enseñanza de la física mediante fislets que incorporan mapas conceptuales híbridos. Apertura, 10(2), 20-35. http://dx.doi.org/10.32870/Ap.v10n2.1335

Moreno, M. N., Font, M. V. y Angulo, V. R. G. (2018). Un estudio sobre la comprensión de las nociones físicas de la mecánica newtoniana: el caso del centro de masa. Revista de Enseñanza de la Física, 30(2), 7-22.

Moreno, M. N., Torres, M. R. de G. y Zúñiga, M. S. C. (2019). Enseñanza de Ecuaciones Diferenciales Ordinarias de primer grado mediante Mapas Conceptuales Híbridos. Investigación e Innovación en Matemática Educativa, 4(1), 2-17.

Moreno, M. N., Zúñiga, M. S. C. y Tovar, R. D. A. (2018). Una herramienta gráfica para la resolución de problemas de cinemática. Latin-American Journal of Physics Education, 12(4). Revista electrónica sin paginación.

Serway, A. y Jewett, W. (2008). Física para Ciencias e Ingeniería. México: Cengage Learning Editores S. A. de C. V. 


\title{
Analysis of the resolution of a kinematics problem using the hybrid conceptual map
}

\author{
Nehemías Moreno Martínez \\ Facultad de Ciencias, Universidad Autónoma de San Luis Potosí, San Luis Potosí, México \\ nehemias.moreno@uaslp.mx \\ Luis Enrique Hernández Zavala \\ Centro de Investigación y de Estudios Avanzados del Instituto Politécnico Nacional, Ciudad de México, México \\ luisenri.hernadez@cinvestav.mx
}

Eduardo Carlos Briceño Solís

Unidad de Matemáticas, Universidad Autónoma de Zacatecas, Zacatecas, México.

ecbs74@gmail.com

The hybrid conceptual map technique is interpreted from an adaptation to school physics of some theoretical constructs of the ontosemiotic approach from Mathematics Education, more particularly, the constructs of mathematical object, semiotic function, practice, processes and perspectives were adapted. The adaptation starts from the idea that physical objects and physical-mathematical objects intervene in the resolution of a physical problem, the former are thought of as abstractions or idealizations of the facts, concrete objects or physical entities that are linked to the experimental results, while the latter are modeled by means of relationships of significance established between some physical objects and mathematical objects understood in the sense of the ontosemiotic approach.

The hybrid conceptual map or hybrid map is built from the oral and written production made by the subject, novice or expert, that solves a certain physical problem. The map thus constructed is called epistemic if it corresponds to the production of an expert and cognitive if it corresponds to that of a novice. The interpretation of the map from this theoretical adaptation mentioned above allows to graphically analyze the physical-mathematical activity of the subject involved in the resolution of a physical problem.

The interpretation of the hybrid map made it possible to adress the problem of the use of the notion of representation as internal/external in the study of the physical-mathematical activity involved in solving physical problems and, alternatively, the objective of investigating the notion of representation when considering it in terms of two dual perspectives ostensive/non-ostensive and cognitive/epistemic adapted from the ontosemiotic approach. It was hypothesized that the internal/external perspective triggers certain ambiguities, for example, an external representation allows students to observe and distinguish various epistemological elements, however, it does not say anything about what would be the internal representation corresponding to said epistemological elements represented externally.

Through a descriptive case study, the interpretation of the hybrid map made it possible to study the practices of solving a parabolic motion problem carried out by a teacher and two of her university physics students, Ana and Bruno. The interpretation of the hybrid maps allowed to graphically represent and analyze the physical and physical-mathematical objects that intervene in the resolution of the physical problem posed, the organization and connection of the objects that participate in the resolution practice system as well as the processes carried out by the subjects.

The results show that the teacher solves the problem correctly through the realization of a set of cognitive processes (reading comprehension, visualization, idealization, particularization, argumentation, meaning and materialization) necessary to produce physical-mathematical knowledge in the problem solving process.

The student Ana obtained correct solutions to the problem, however, she performed blind operativism by attending to superfluous aspects of the problem. The hybrid map, by showing the connections between the objects that participate in solving the problem, leads to reformulate blind operativism as an inadequate realization or the absence of some of the cognitive processes instead of an absence of relationship between the conceptual and the procedural. The student Bruno carried out the same set of processes as the teacher, however, he solved the problem posed incorrectly due to the lack of adequate prior knowledge.

The interpretation of the hybrid maps allows us to conclude that the physical-mathematical activity can be described in a more adequate way by considering the notion of representation in terms of two facets or dual perspectives, cognitive/epistemic and ostensive/non-ostensive, instead of interpreting it only as internal/external. 